\title{
Kidney fibrosis in disease and aging - The stage and some key actors
}

\author{
Rui Alves
}

Nephrology Department, Faculty of Medicine of Coimbra, Coimbra, Portugal

Nephrology Department, Hospital and University Centre of Coimbra, Coimbra, Portugal

\section{INTRODUCTION}

Deepening the understanding of the pathophysiological mechanisms of disease in all organ systems has always been the cornerstone of progress and one of the greatest challenges of modern medicine. The remarkable advances in biotechnology over the past two decades have yielded explanations for many of the cellular and molecular mechanisms implicated in disease and aging. Unexplored paths have been opened for more specific diagnosis and more selective therapy, and also for adopting the best strategy to delay the wear and tear that advancing age involves.

Conceptually, disease progression and tissue aging culminate in destruction with cell death and replacement fibrosis. This terminal phenomenon has often been considered a marginal and demotivating event because it appears to be unchanging and almost always inexorable, to which research would add little. However, from the 1980s, renal fibrosis began to be looked at from two sides, an example of a Janus-like phenomenon - as an enemy, or friend.

There are numerous examples where the repair process through fibrosis is crucial for organ protection, function and survival (i.e. surgical scar, myocardial infarction scar). But as it is a crude repair, the impact on function will depend on the organ and the extent of the injury. In the case of the kidney, it represents the final pathway common to most chronic and progressive nephropathies and depends on the invasive assault, part affected, and duration and intensity. The injury may be dramatic, with irreversible destruction of essential microstructures and irreversible loss of function, or slow, with progressive loss, as in chronic kidney disease and renal aging.

This biological and ancient form of fibrogenesis-based repair has always been regarded as a point of no return, but it is now the reason for much research into the regenerative potential of tissues. In fact, nature has examples of living beings in which repair simply means full regeneration; examples being some amphibians that can reconstruct body segments after amputation. This context serves to demonstrate the unfathomable potential ability of stem cells (tissue, bone marrow and others) to multi-differentiate and repair mature tissues, and questions the point of no return, paving the way for the study and development of regenerative medicine.

Renal aging concerns structural changes generally known as nephrosclerosis (i.e. decreased size and number of glomeruli, tubulointerstitial changes, thickening of the glomerular basement membrane, glomerulosclerosis). These modifications have not been shown to correlate with a decline in the glomerular filtration rate (GFR), age, albuminuria, family history of end-stage renal disease (ESRD), body mass index, serum cholesterol or uric acid. And we know that many of the mechanisms of kidney injury, whether genetic, immune mediated or caused by toxins, are also present in the aged kidney, albeit with gradual onset ${ }^{1}$.

Thus, although still far from proven, when we try and examine the myriad mechanisms in fibrosis repair and regeneration, we can find a parallel between the complicated process of cell senescence/aging, and the pathogenesis of kidney disease, since they coincide in many of the biological phenomena implicated in fibrosclerosis. These conjectures raise a number of questions: Is fibrosis the final and irreparable event in disease and aging? Does it depend on the disease and the individual? What cells and mechanisms are involved? Is it possible to modulate it or make it regress? is regeneration possible? Or is it simply a point of no return?

At the crossroads of the lively debate on these issues is the primary goal of selectively inhibiting or slowing down fibrosis in disease and aging. Admittedly, many of the indirect mechanisms leading to changes in the extracellular matrix turnover (i.e. inflammation, oxidative stress, renin-angiotensin-aldosterone system (RAAS) activation), and even various drugs that can interfere at that level are already known. But we still have to know for certain which are the key genes, molecules and cells in this fascinating biological carousel. In fact, most advances are the result of testing in experimental models, which, although valuable research aids, have limitations that constrain the linear extrapolation of the findings to the human kidney.

\section{EXTRACELLULAR MATRIX TURNOVER IN DISEASE - Molecules, cells and genes}

Fibrosis is characterized by the excessive and disorganized deposition of various components of the extracellular matrix, particularly collagen. This deposition can occur at the glomerular, vascular and-or tubulointerstitial level, depending on the primary site of the injury. As noted, the best aids to this study have been experimental models of inflammation and fibrosis (i.e. nephrotoxic serum nephritis (NSN) model; anti-GBM disease model and anti-Thy-1.1 podocyte model in focal glomerulosclerosis). But others can be indicated, such as unilateral ureteral obstruction (UUO), a model of human obstructive nephropathy, and transgenic models with disruption of the COL4A3 gene in the case of Alport syndrome, or with variants of the Wilm's tumor gene 1 (WT) expression, whose presentation can range from crescentic glomerulonephritis to mesangial sclerosis². 
The architectural organization of the nephron, with an intricate network of microvessels and tubules, maintains the glomerulotubular physiological balance but also permits the diffusion of glomerular disease into the tubulointerstitial compartment, exposing the tubular and interstitial epithelium to a large number of filtered molecules with inflammatory and pro-immunological activity. It should be noted that interstitial involvement is still considered the best histological predictor of declining renal function in glomerular diseases and type 1 diabetic nephropathy.

Depending on the duration and intensity of the initial insult, inflammatory cell infiltration is accompanied by a cascade of phenomena including fibroblast activation, monocyte/macrophage infiltration, and epithelium-mesenchymal transition (EMT), in which activated tubular cells produce pro-inflammatory molecules, including chemotactic cytokines (i.e. monocyte chemoattractant protein (MCP-1); regulated upon normal activation $T$ cell expressed and secreted (RANTES) and chemotactic activation of C3 and C5). Leukocyte adhesion (ICAMs) and vascular adhesion molecules (VCAMs) also play an important role in the recruitment and migration of mononuclear cells into the glomeruli and interstitium, with the production of other inflammatory and pro-fibrogenic molecules including reactive oxygen species $(\mathrm{ROS})^{1,3}$.

Far from exhausting the extreme complexity of these mechanisms, the importance of several key fibrogenesis mediating molecules has been demonstrated, such as transforming growth factor (TGF), angiotensin 2 (AngII), connective tissue growth factor (CTGF), platelet derived growth factor (PFGF) and endothelin-1. TGF $\beta 1$ can be produced by resident cells and infiltrating leukocytes and their activity is closely related to sequential and intracellular signaling of the Smad family of proteins (stimulators - Smad2, Smad3), or inhibitors (Smad 7). These inflammatory stimuli activate mesangial cells, fibroblasts and tubular epithelial cells by inducing the synthesis of large amounts of extracellular matrix, which can reach the three compartments of the kidney 4 .

It should be noted that even in the absence of injury or disease, the extracellular matrix exhibits a physiological and dynamic turnover regulated by the balance between matrix-degrading enzymes (metalloproteinases - MMP) causing cleavage of components, and their inhibitors (tissue inhibitor metalloproteinases - TIMP). These enzymes are produced by intrinsic glomerular cells, tubular epithelial cells and macrophages, and in fibrosis the disorganized enlargement of the extracellular matrix is a consequence of disruption of this balance ${ }^{3}$.

It is very interesting to note that some signaling pathways of some critical genes are simultaneously implicated in nephrogenesis and experimental renal fibrosis (i.e. Wnt, Hedgehog, Notch). And those signaling pathways may exhibit variable behavior depending on the stimuli and the cellular environment in which they are activated.

Interstitial pericytes and fibroblasts are important targets of tubular Wnt signaling pathway ligands in the activation of myofibroblasts, regardless of inflammation, which means that inflammatory mechanisms might not be required for the development of fibrosis. It further means that tubular secretion of these ligands is sufficient to cause spontaneous interstitial fibrosis by paracrine signaling between the tubular epithelium and the interstitial myofibroblasts ${ }^{5}$.
Other experimental studies have shown the involvement of Notch pathway signaling in kidney embryogenesis, notably in controlling cell differentiation. Epithelial de-differentiation in chronic kidney disease (CKD) has been shown to induce activation of the Notch pathway, and both are required for tubulointerstitial fibrosis. Induction of this pathway activates cell proliferation and inhibition of cell differentiation, with activation of the transcriptional program of the epithelium-mesenchymal transition, in which transcription factors such as Snail1 appear to play an important role. In this context, the mesenchymal epithelium transition means that the epithelial cell has the potential to secrete pro-fibrotic factors that activate interstitial myofibroblasts without turning into myofibroblasts, as had previously been suggested ${ }^{6}$.

\section{MYOFIBROBLAST - The culprit (?)}

Tubulointerstitial fibrosis is the paradigm of aging and chronic kidney disease, regardless of the cause. The interstitial fibroblast, glomerular mesangial cell, and vascular wall smooth muscle cells are phenotypically similar and may acquire smooth muscle characteristics when activated. These cells are recognized by the renewed synthesis of alpha smooth muscle actin ( $\alpha$ SMA), and thus called myofibroblasts, which play a critical role in fibrosis progression. As they proliferate, they synthesize extracellular matrix components, they contract and the effects of tension on adjacent cells contribute to reorganizing the matrix and increasing density.

Over the years, published works have shown there is great controversy as to the nature of these cells, and especially their origin (progeny). A better knowledge of the molecular mechanisms conducing to the appearance of differentiated myofibroblasts in each pathological situation will be useful for the understanding of fibrosis development in different organs. Genetic lineage analysis techniques have contributed to disclose the various potential myofibroblasts progenitor pools. The most recent evidence indicates resident stromal cells, such as pericytes (Gli1 + ), perivascular fibroblasts, or mesenchymal stem cells as the most likely source of myofibroblasts. These could account for about $55 \%$ of the total myofibroblast pool and can be a potential therapeutic target. But other cells, derived from bone marrow (macrophages and fibrocytes), endothelial cells, and epithelial cells, through the epithelial-mesenchymal transition (EMT) process, could be implicated. In the case of epithelial cells, however, the most recent studies point to a reduced participation ${ }^{7}$.

According to the same studies, the remaining $45 \%$ of myofibroblast progenitor candidates could originate from the bone marrow and endothelium by transitioning these cells to the mesenchymal phenotype when exposed to TGF $\beta$. Other researchers concluded that marrow-derived cells are the progenitors of myofibroblasts, and include mesenchymal stromal cells (MSCs) and fibrocytes (CD45 +, CD34 +), the latter derived from circulating monocytes ${ }^{8}$.

Despite the progress, doubts remain as to the distinction of the main subtypes of myofibroblast progenitors, and the importance of their in vivo contribution, because much of the known results have been obtained in vitro. New technologies, in particular single-cell RNA sequencing, are needed to better define the hierarchy of this interstitial cellular intervention. 


\section{EXTRACELLULAR MATRIX TURNOVER IN AGING - Genes, cells and molecules}

It is important to distinguish the mechanisms of genetic, immune or toxic origin underlying chronic kidney disease (CKD) from the gradual changes typical in a healthy elderly kidney, but this is limited by the scarcity of studies and the difficulty of extrapolation to the human kidney. Changes such as cell senescence, fibrosis, vascular rarefaction and glomerular loss are found in aged kidneys and CKD. Genome-wide association studies (GWAS) have equated the participation of 50 genetic loci in chronic kidney disease and in the evolution of estimated glomerular filtration rate (GFRe). Although no critical link has been identified with known pathways of aging in GWAS, research in this area suggests that over $80 \%$ of heredity in aging could be explained by common genetic variants. Several genetic loci have been studied, such as Wnt signaling, which is key during nephrogenesis, but also with a pro-fibrotic component through the expression of a single nucleotide polymorphism (SNP) in the intron of the WNT7A gene that can be experimentally inhibited ${ }^{9}$.

The intronic region of another gene, NFKB1, promotes the transcription of age-associated genes in the human kidney, and a link between mutations in TLR4 or IL-1 and kidney damage, or ESRD, respectively, in which the hypothesis that this impact is related to a pericyte-mediated inflammation is advanced ${ }^{10}$.

The Klotho gene is known to play an important role in modulating several age-related pathways, notably by decreasing epithelial cell senescence in response to oxidative stress, and attenuating fibrosis in the UUO model. Its performance also includes fibroblast growth factor (FGF) suppression and Wnt signaling modulation, the expression of which increases as Klotho levels decrease with age, promoting fibrosis and vascular calcification ${ }^{11}$.

Another important pathway in this domain is the renin-angiotensinaldosterone system (RAAS) axis, whose activation is also mediated by Wnt gene signaling. Angiotensin II (AT2) levels increase with age promoting increased extracellular matrix with fibrosis, and increased oxidative stress ${ }^{12}$. As is well known, oxidative stress is an important factor in the expression of the aging phenotype, which is accompanied by decreased tissue antioxidative capacity (superoxide dismutase (SOD), catalase and glutathione (GSH) reductase). In this context it has been shown that sirtuins (antioxidant molecules) decrease with age and in their absence there is a poorer capacity for oxidation response, with increased inflammation, fibrosis and apoptosis, creating the conditions for accelerated aging ${ }^{13}$.

\section{CELL SENESCENCE AND HYPOXIA}

Chronic kidney disease and fibrosis affect $50 \%$ of the population over 70 years of age, and it is difficult to assess the quantitative significance of the senescence component with the decline of renal function over time.

Here, too, the tubulointerstitial cross talk plays an essential part in the progression of fibrosis. After an injury, whether the onset is sudden or slow, the epithelial cell de-differentiates with overregulation of inflammatory proteins, oxidative stress, telomere shortening and DNA damage. Taken together, these processes stimulate the overregulation of cyclin-dependent kinase inhibitors that cause cell arrest in the G2/M phase. But senescent cells do not behave amorphously; on the contrary, they adopt a senescence-associated secretor phenotype, which resists apoptosis and secretes many paracrine-acting proinflammatory and pro-fibrotic mediators (cytokines, TGF- $\beta$ and proteases $)^{14}$. Inhibition of the proximal tubule senescence phenotype (mutations in INK4 - cyclin-dependent kinase inhibitors) has been shown to be accompanied by less interstitial fibrosis and tubular atrophy, with increased proliferation in an ischemia-reperfusion model, and improved glomerulosclerosis related to age.

Paradoxically, when pharmacological quiescence is induced in acute injury associated with post-ischemic inflammation, the epithelial cell cycle arrest, through inhibition of cyclin-dependent kinase (CDK4/6), allows DNA repair, reduction of apoptosis and of cell senescence itself $^{15}$.

The importance of renal blood flow $(400 \mathrm{ml} / 100 \mathrm{~g})$ is recognized, which far exceeds the level of other vascular beds, such as the heart, brain or liver, making the kidney more susceptible to harmful agents and other circulating substances. In fact, the kidney is highly dependent on blood perfusion with sufficient oxygen content to fulfill the metabolic and nutritional functions of the tissue. The "chronic hypoxia hypothesis", launched two decades ago, postulates that tubulointerstitial hypoxia is a determining factor in the onset and progression of chronic kidney disease, and several experimental models have demonstrated its presence ${ }^{16}$. More recently, it has been observed that inducible hypoxia factor (HIF-1a) is overregulated in hypoxic epithelial cells and regulates signaling of pro-fibrotic pathways. Therefore, it is not difficult to assume that loss of peritubular capillaries in acute and chronic injury, and particularly in aging, helps to perpetuate cellular ischemia. The aforementioned role of perivascular cells $(\mathrm{Gli} 1+)$ and their potential to transform into myofibroblasts that generate interstitial fibrosis is framed herein, but there might be other mechanisms ${ }^{17}$. Experimental studies have proposed another way in which these cells could intervene by showing that their ablation was accompanied by endothelial lesion with capillary rarefaction and hypoxia, and induction of tubuloepithelial transition. It seems therefore, that these perivascular mesenchymal-like cells could be important in stabilizing intrarenal vasculature, and that pericyte-endothelium interaction could also be a therapeutic target in fibrosis. These observations underline the importance of pro- and anti-fibrotic vasculogenesis, and motivate the development of models in which angiogenic regulation and the stimulation of neovasculogenesis might have a protective effect on aging.

\section{FINAL COMMENT}

The extraordinary functional complexity of physical and chemical mechanisms of the kidney, based on its unique anatomical-physiological structure, makes it a particularly interesting organ to study in the context of the near ubiquitous phenomenon of fibrosis. In disease, aging, and regeneration, the extraordinary potential and plasticity of the more than 20 different types of cell that make it up underscore the importance of the tubular and interstitial epithelium as manager 
sites for numerous signaling and cross-communication pathways between tubules, vessels and glomeruli.

Kidney disease and aging share many pathophysiological mechanisms, mobilizing a myriad of inflammatory and fibrogenic molecules, that converge to stimulate the production of extracellular matrix components. The origin of the main target cell is still under discussion, and over the past twenty years, although the origin of myofibroblast has been transferred to periadventitial cells, the tubular epithelium remains an essential and auspicious leading factor in understanding the mechanisms of fibrogenesis. Proof of this lies in more recent in vivo studies that have shown that myofibroblasts can be directly reprogrammed into tubular cells, making this a possible strategy in regenerative medicine.

In addition, we are facing genetics in the overall control of inflammatory response and cell survival mechanisms, which are determinants of disease and aging. Somewhat disappointingly, the findings have not yet made it possible to isolate key and unambiguous actors (genes) that follow a predictable pattern of behavior to proactively intervene and protect tissue degeneration.

Notwithstanding all the difficulties in obtaining clear answers to our ongoing questions, it is undisputed that the general measures to prevent and treat the major diseases of our time are already on the right track, but it is crucial to continue the research work in this fascinating area of fibrosis, to be truly able to revolutionize the future of humankind.

Disclosure of potential conflicts of interest: none declared

\section{References}

1. O'Sullivan ED, Hughes J, Ferenbach DA. Renal aging: causes and consequences. J Am Soc Nephrol 2017; 28: 407-420

2. Guo JK, Menke AL, Gubler MC, Clarke AR, Harrison D, Hammes A, et al. WT1 is a key regulator of podocyte function: reduced expression levels cause crescentic glomerulonephritis and mesangial sclerosis. Hum Mol Genet 2002; 11: 651-659.

3. Eddy AA. Molecular basis of renal fibrosis. Pediatr Nephrol 2000; 15:290-301.

4. Ruiz-Ortega M, Ruperez M, Esteban V, Rodriguez-Vita J, Sanchez-Lopez E, Carvajal G, et al. Angiotensin II: a key factor in the inflammatory and fibrotic response in kidney diseases. Nephrol Dial Transplant 2006; 21: 16-20.

5. Maarouf $\mathrm{OH}$, Aravamudhan $\mathrm{A}$, Rangarajan $\mathrm{D}$ et al. Paracrine Wnt1 drives interstitial fibrosis without inflammation by tubulointerstitial cross-talk. J Am Soc Nephrol 2015; 27: 781-790.

6. Grande MT, Sánchez-Laorden B, López-Blau C, et al. Snail1-induced partial epithelial-to-mesenchymal transition drives renal fibrosis in mice and can be targeted to reverse established disease. Nat Med 2015; 21: 989-997.

7. Kramann R, Schneider RK, DiRocco DP, et al. Perivascular Gli+ progenitors are key contributors to injury-induced organ fibrosis. Cell Stem Cell 2015; 16: 51-66.

8. Niedermeier M, Reich B, Rodriguez Gomez M, et al. CD4+Tcells control the differentiation of Gr1+ monocytes into fibrocytes. PNAS 2009; 106: 17892-17897.

9. Wuttke M, Kottgen A. Insights into kidney diseases from genome-wide association studies. Nat Rev Nephrol 2016; 12: 549-562.

10. Leaf, IA, Nakagawa S, Johnson BG et al. Pericyte MyD88 and IRAK4 control inflammatory and fibrotic responses to tissue injury. J Clin Invest 2017; 127: 321-334.

11. Bian A, Neyra JA, Zhan M, Hu MC. Klotho, stem cells, and aging. Clin Interv Aging 2015; 10: $1233-$ 1243.

12. Benigni A, Cassis P, Remuzzi G: Angiotensin II revisited: new roles in inflammation, immunology and aging. EMBO Mol Med 2010; 2: 247-257.

13. Martin R, Fitzl G, Mozet C, Martin H, Welt K, Wieland E. Effect of age and hypoxia/reoxygenation on mRNA expression of antioxidative enzymes in rat liver and kidneys. Exp Gerontol 2002; 37: 1481-1487.

14. Sturmlechner I, Durik M, Sieben CJ, Baker DJ, van Deursen JM. Cellular senescence in renal ageing and disease. Nat Rev Nephrol 2017; 13: 77-89.

15. DiRocco D, Bisi J, Roberts $P$, et al. CDK4/6 inhibition induces epithelial cell cycle arrest and ameliorates acute kidney injury. Am J Physiol Renal Physiol 2013; 306: 379-388.

16. Fine LG, Orphanides C, Norman JT. Progressive renal disease: the chronic hypoxia hypothesis. Kidney Int. 1998; 65: S74-78.

17. Kramann R, Wongboonsin J, Chang-Panesso M, Machado FG, Humphreys BD. Gli1+ pericyte loss induces capillary rarefaction and proximal tubular injury. J Am Soc Nephrol 2017; 28: 776-784.

\section{Correspondence to:}

Rui Alves

Nephrology Department, Centro Hospitalar e Universitário de Coimbra, Coimbra, Portugal

E-mail: ralves@fmed.uc.pt 